\title{
A Recipient and Donor Both Have COVID-19 Disease. Should We Perform a Liver Transplant?
}

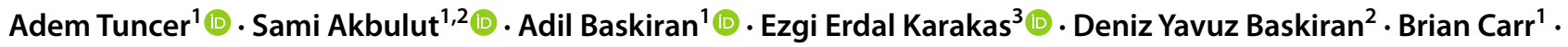 \\ Sezai Yilmaz ${ }^{1}$ (1)
}

Accepted: 19 January 2021 / Published online: 18 February 2021

๑) Springer Science+Business Media, LLC, part of Springer Nature 2021

\begin{abstract}
Coronavirus 2019 (COVID-19) is a new infectious disease that continues to spread globally. There is growing concern about donor-induced transmission of Coronavirus 2 (SARS -CoV-2). For liver transplantation, the COVID-19 PCR test is routine, in addition to epidemiological history and clinical and radiological examination $24-48 \mathrm{~h}$ before surgery. One of the liver transplant candidates was found to be infected with COVID-19, as well as the planned donor candidate. Since COVID-19 will be a high-risk operation for both the recipient and the donor, the operation was postponed by giving medical treatment. After the treatment and quarantine process was over, the patient and the donor then had a negative COVID-19 PCR test and the patient received a living donor liver transplant. We present a case of donor and recipient who initially both tested positive for COVID-19. This liver transplantation scenario has not previously been reported in the literature.
\end{abstract}

\section{Introduction}

In December 2019, a new Coronavirus outbreak was detected in Wuhan, China. This virus was named severe Acute Respiratory Syndrome Coronavirus 2 (SARS -CoV-2), and the disease it caused was named coronavirus disease 2019 (COVID-19). The virus was declared a pandemic by the World Health Organization on March 11, 2020, as the transmission of the virus quickly found in millions of people worldwide. The clinical spectrum of COVID-19 varies widely, from asymptomatic carrier state to multisystemic, fatal pneumonia affecting the lung, liver, and heart [1]. The severity of the COVID-19 disease is directly related to the patient's age and the presence of comorbidities [2].

The impact of COVID-19 disease on liver transplant programs and recipients is still not fully understood. The risk of donor-borne transmission, the reliability of diagnostic tests, and the effect of immunosuppression are still unclear. Liver

Adil Baskiran

dr.adil.baskiran@gmail.com

1 Department of Surgery, Liver Transplant Institute, Inonu University, 44280 Malatya, Turkey

2 Department of Public Health, Faculty of Medicine, Inonu University, 44280 Malatya, Turkey

3 Department of Infection Disease, Faculty of Medicine, Inonu University, 44280 Malatya, Turkey transplant programs have been deeply affected by COVID19 caused by SARS-CoV-2. Solid organ transplant programs have declined even in areas with low COVID-19 prevalence [3]. The American Society of Transplant Surgeons (ASTS), published on March 24, 2020, recommended not to transplant from donors infected with COVID-19 [4]. This protocol is universally designed on all donors and transplant candidates, whether the COVID-19 tested person is symptomatic or not. According to this protocol, the patient should be evaluated using a history, physical examination, PCR test, computed tomography (CT), and transplant infectious disease doctor consultation. Thanks to these decisions, it minimizes risks for all patients and healthcare professionals [5]. There is a theoretical risk of transmission of COVID-19 through transplantation, but donor-borne transmission has not been reported to date. Proinflammatory cytokines may play an important role, especially in the more severe form of COVID-19. Because immunosuppressive drugs suppress proinflammatory cytokines, transplant recipients may be protected to some extent from SARS-CoV-2 infection and associated complications [6].

We routinely evaluate donors and recipients with radiological, clinical, epidemiological, and COVID-19 PCR testing before performing a living donor liver transplant (LDLT). The donor and recipient test results of the COVID-19 PCR test were positive in a recipient and donor pair who we prepared for LDLT, and the patient had a Model for End-stage Liver Disease 
(MELD) score above 19. After the COVID-19 treatment and quarantine process were completed, the recipient candidate was successfully transplanted 21 days later without decompensation. Close follow-up of donor and recipient, who were without symptoms of postoperative COVID-19, was uneventful. In the literature, we did not find any cases that were positive for the preoperative COVID-19 PCR test, recovered after treatment, and then underwent LDLT. We wanted to present this rare case.

\section{Case Report}

A 34-year-old male donor candidate, whose medical history and physical examination were unremarkable, and his father, a 60 years old with cryptogenic liver failure and a MELD score above 19, applied to our institute to have a live donor liver transplant. According to our routine blood tests, Multislice Liver Tomography, and MR results, there was no obstacle for the donor candidate to become a donor. According to our calculation, the right lobe of the donor liver was $770 \mathrm{cc}$ and the remnant was $30 \%$. When the COVID-19 PCR test was positive in the combined nasopharyngeal and oropharyngeal swab we received from the donor, he was quarantined and treatment was started. Control PCR test became negative after 11 days. Meanwhile, the COVID-19 PCR test was positive in the combined nasopharyngeal and oropharyngeal swab we made on the recipient candidate. The recipient candidate was also treated and quarantined. Control PCR test given after 11 days was negative. Recipient and donor candidates, whose treatments and quarantine processes were completed, were re-evaluated by laboratory tests and clinically. Considering the toxic effect of COVID-19 on donor and recipient liver tissue, follow-up surveillance continued for another 10 days. The fact that COVID-19 is ACE-2 receptor focus especially in bile duct cells, a hepatitis picture that may be caused in this situation was taken into consideration. Approximately 21 days later, the COVID-19 PCR test taken from both donor and recipient was negative. In addition, there was no pulmonary involvement radiologically. (Figures. 1 and 2) In the laboratory, no pathology was found in the liver function tests of the donor.

LDLT was successfully performed in our center. The donor and recipient were taken to the intensive care unit, and the donor was extubated postoperatively. During the intensive care follow-up, the donor's pulse, respiratory rate, saturation, and blood pressure remained within normal ranges. In our center, $4 \mathrm{~g}$ ampicillin + sulbactam antibiotic prophylaxis, which is routinely applied for donors on the postoperative 0th day, was initiated on the 1st day, enteral feeding was initiated, and 4000 IU low molecular weight heparin (LMWH) was administered for deep vein thrombosis prophylaxis and mobilized. There were no postoperative complaints of cough, sputum, fever, and fatigue, and the donor, whose radiological images were also normal (Fig. 3), did not need additional antibiotics. Other blood tests of the donor were not different from other donors. Ferrittin: 176.2 (normal range of 22-322), D-Dimer: 4.4 (0-0.55), IL-6: 33 (0-7), Fibrinogen: 228 (150-350), CRP: 4 (0-0.35), PCT:
Fig. 1 Preoperative thoracic CT section of the recipient

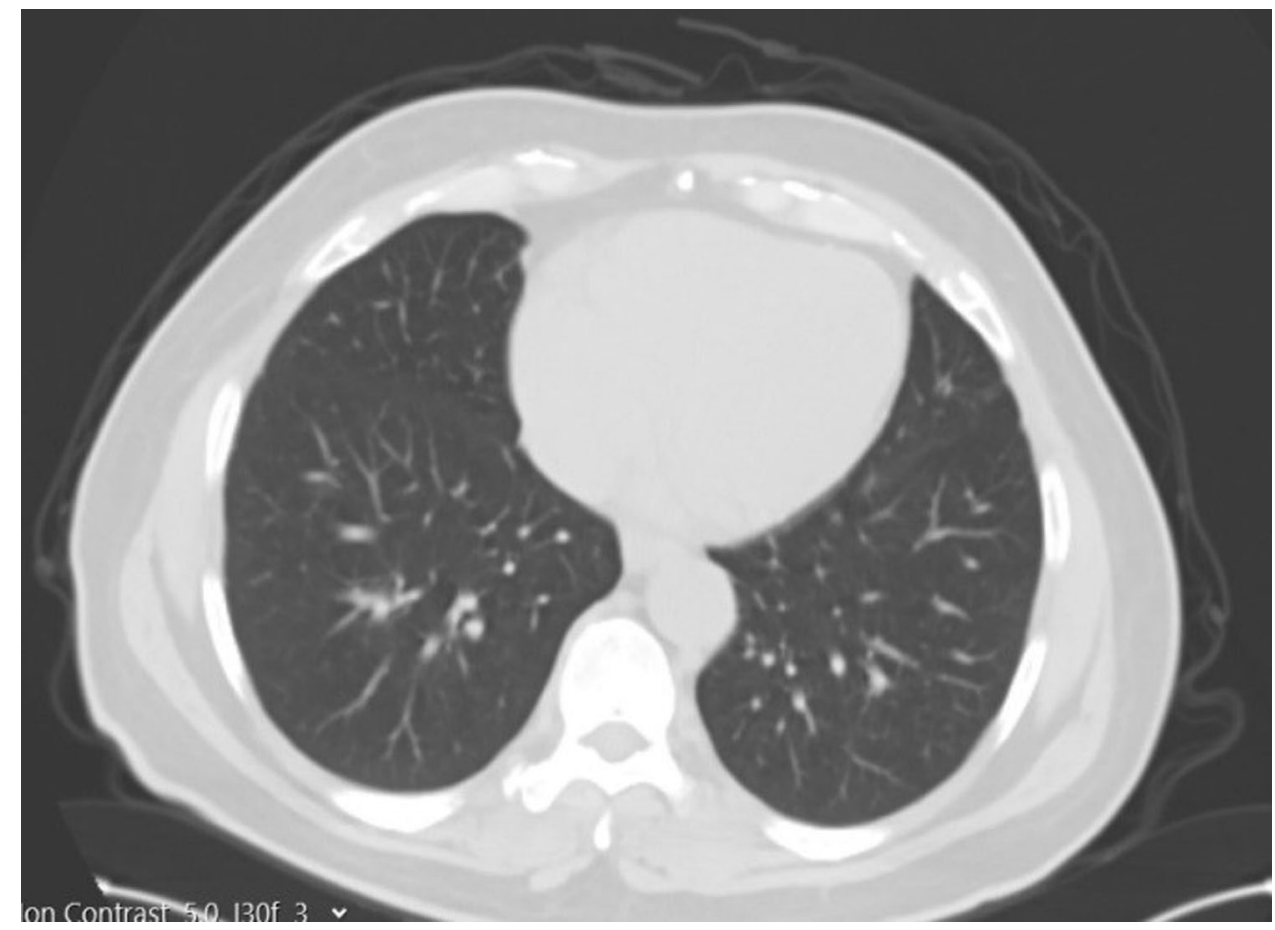


Fig. 2 Preoperative thoracic CT section of the donor

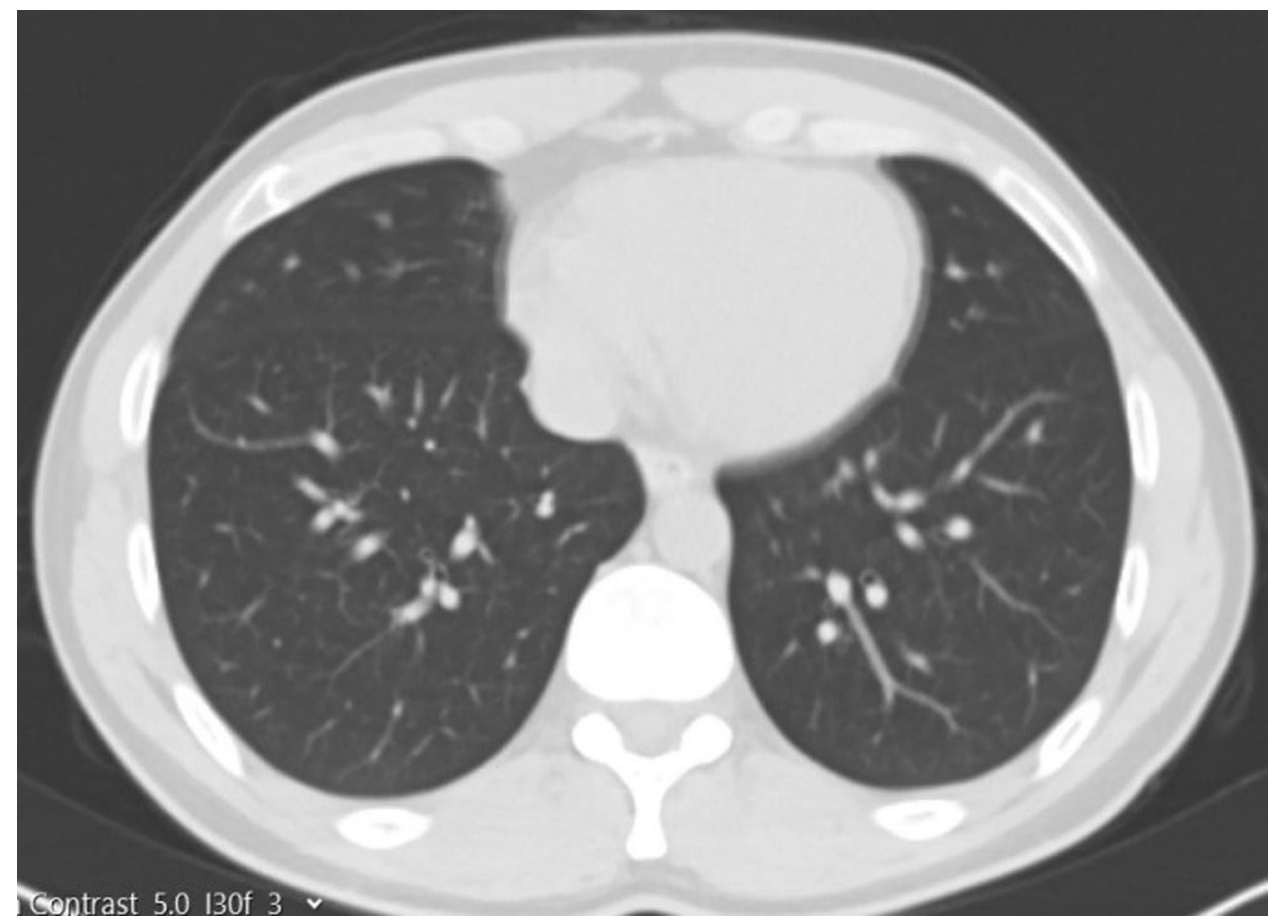

0.3 (0-0.5), Lymphocyte: 0.84 . On the postoperative 14 th day, the donor was discharged without any problem.

Recipient postoperative intubation and organ transplantation with noradrenaline support were taken to the intensive care unit. In our center, a total of $8 \mathrm{gr}$ of ampicilin+sulbactam antibiotic prophylaxix, which is routinely applied for recipients, was initiated on postoperative 0 and 1 days. On postoperative day 1 , the recipient was extubated, enteral feeding was initiated, and the patient was mobilized. There were no complaints of cough, sputum, fever, and weakness, and there was no need for additional antibiotics for the recipient whose radiological images were also normal (Fig. 4). Immunosuppressive drugs routinely used in our center to prevent liver rejection are used in the same doses. These are $2 \times 100 \mathrm{mg}$ methylprednisolone and $2 \times 1000 \mathrm{mg}$ mycophenolate mofetil on postop $1 \mathrm{st}$
Fig. 3 Donor postoperative thoracic CT section. Postoperative thoracic CT section of the recipient on the right

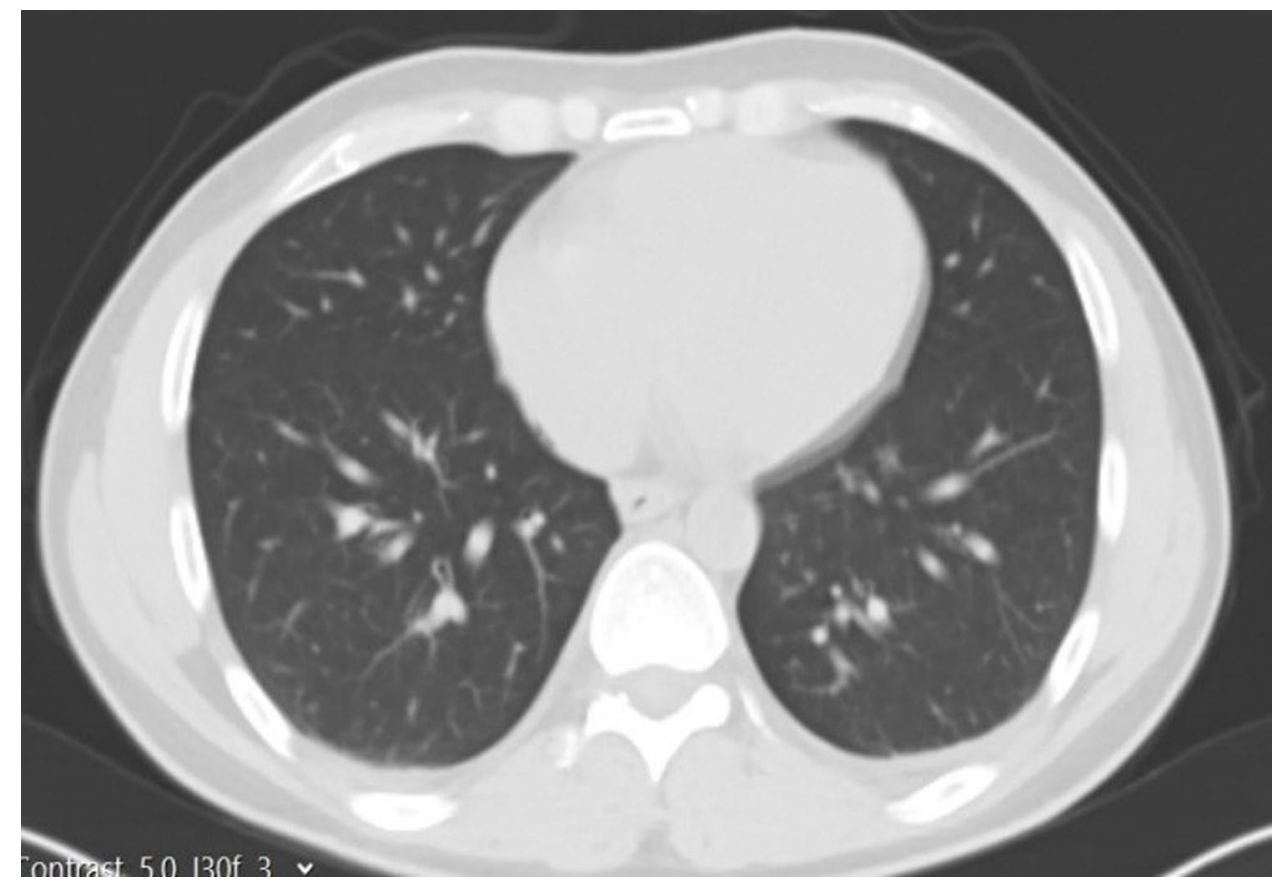


Fig. 4 Postoperative thoracic CT section of the recipient

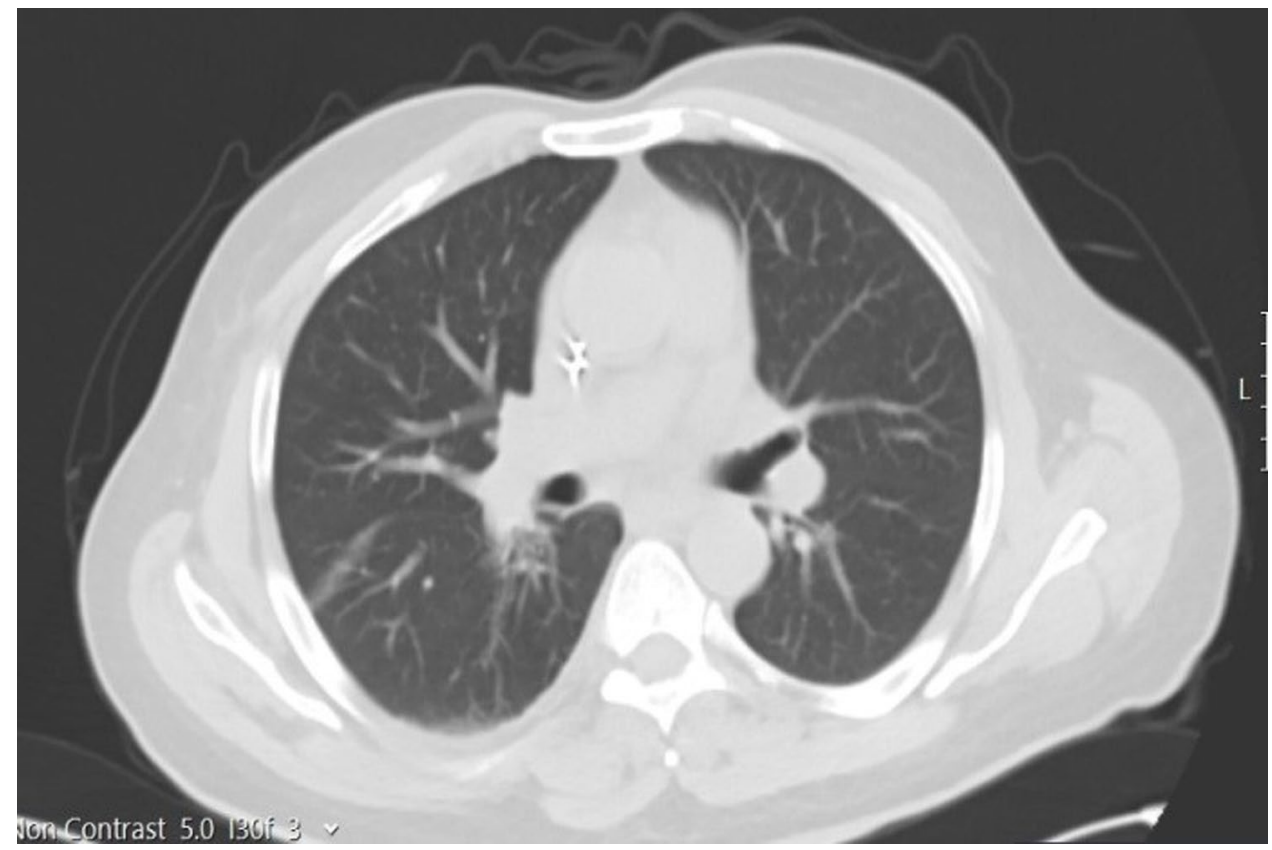

day and $2 \times 1 \mathrm{mg}$ Tacrolimus started on the 3rd day. The recipient, whose blood tests and Doppler results were normal, was taken to the service on the 3rd postoperative day. Ferrittin: 559 (normal range 22-322), D-Dimer: 0.3 (0-0.55), IL-6: 9.7 (0-7), Fibrinogen: 176 (150-350), CRP: 2.2 (0-0.35), PCT: 1.6 (0-0.5), Lymphocyte: 0.44. The follow-up of the recipient on the postoperative 16th day continues without any problem.

\section{Discussion}

With the announcement of COVID-19 as a pandemic, many studies have been carried out on the precautions to be taken by health institutions and the measures to be followed. Recent studies have shown that hospitalizations should be reduced and elective surgery should be delayed as much as possible [7]. However, postponing liver transplantation operations may cause an increase in the number of transplantation waiting lists, worsening, or even death of patients waiting for transplantation. Patients with end-stage liver failure and high MELD are at risk of decompensation during the waiting list. If the transplantation surgery of patients with malignancy is delayed, they may lose the chance of surgery due to tumor size increase and metastasis. We postponed the operation of our patient, whose COVID-19 PCR test was positive, with close follow-up, and then we performed the transplant surgery immediately after the donor and recipient tests without COVID-19 symptoms were negative. We continued to follow-up closely after the surgery; there were no symptoms and laboratory tests in favor of COVID-19. Both donor and recipient Ferritin, D-Dimer, Interleukin-6,
CRP, PCT, Fibrinogen, and Lymphocyte levels were in the normal range.

The vast majority of recent studies argue that transplant operations should be continued despite the pandemic [8, 9]. In the study by Huang et al., a higher risk for COVID19 was reported for solid organ transplant recipients using immunosuppression drugs, thus leading to delay in transplantation surgeries [10]. Aslan et al. reported no relationship between immunosuppressive therapy and COVID-19 mortality [11]. Seminari et al. reported that immunosuppressive therapy can have a protective effect against COVID-19 by reducing the cytokine storm [12]. We applied the immunosuppression protocol that we routinely apply postoperatively for recipient. After immunosuppression, the recipient's Ferritin, D-Dimer, Interleukin-6, CRP, PCT, Fibrinogen, and Lymphocyte levels were normal.

There is no definitive treatment or post-exposure prophylaxis for COVID-19 infection. Recently, hydroxychloroquine, lopinavir, ritonavir, and remdesivir therapy have been suggested as potential treatment options [13]. However, the effectiveness of all these treatments is not fully known. It is not known whether this prophylaxis has any role in preventing SARS-CoV-2 in the recipient from the donor. We think that further studies are needed for this subject. Therefore, we did not give any extra treatment other than the routine antibiotic prophylaxis in our donor and recipient center whose COVID-19 PCR was positive.

As a result, delaying LT procedures during the pandemic period may cause the patients' existing diseases to worsen and the number of patients on the waiting list to increase. Because of the use of an organ taken from a donor infected 
with COVID-19, the possibility of transmission through transplant cannot be ruled out. Donor health is always a priority in LDLT. Therefore, in a donor who has had COVID19 , the donor should not be taken into operation only when the COVID-19 test becomes negative, but also after clinical, radiological, epidemiological, and laboratory evaluation has been done. During the pandemic process, liver transplants can be performed with the precautions taken in cases that cannot be postponed, especially for patients with acute liver failure, decompensated liver failure, and hepatocellular cancer.

\section{Declarations}

Conflict of Interest The authors declare that they have no conflict of interest.

\section{References}

1. Zhou F, Yu T, Du R, Fan G, Liu Y, Liu Z, Xiang J, Wang Y, Song B, Gu X, Guan L, Wei Y, Li H, Wu X, Xu J, Tu S, Zhang Y, Chen H, Cao B. Clinical course and risk factors for mortality of adult inpatients with COVID-19 in Wuhan, China: a retrospective cohort study. Lancet. 2020;395(10229):1054-62.

2. Wu C, Chen X, Cai Y, Xia J, Zhou X, Xu S, Huang H, Zhang L, Zhou X, Du C, Zhang Y, Song J, Wang S, Chao Y, Yang Z, Xu J, Zhou X, Chen D, Xiong W, Xu L, Zhou F, Jiang J, Bai C, Zheng J, Song Y. Risk factors associated with acute respiratory distress syndrome and death in patients with coronavirus disease 2019 pneumonia in Wuhan. China JAMA Intern Med. 2020;180(7):934-43.

3. Di Maira T, Berenguer M. COVID-19 and liver transplantation. Nat Rev GastroenterolHepatol. 2020;17(9):526-8.

4. Organ Retrieval for Transplantation in the COVID-19 Era. ASTS. 2020. https://asts.org/advocacy/covid-19-resources/asts-covid-19-strikeforce/asts-covid-19-strike-force-organ-retrieval-guidance. Accessed April 14, 2020.

5. Galvan NTN, Moreno NF, Garza JE, Bourgeois S, Hemmersbach-Miller M, Murthy B, Timmins K, O’Mahony CA, Anton J, Civitello A, Garcha P, Loor G, Liao K, Shaffi A, Vierling J, Stribling R, Rana A, Goss JA. Donor and transplant candidate selection for solid organ transplantation during the COVID-19 pandemic. Am J Transplant. 2020;20(11):3113-22.

6. Huang C, Wang Y, Li X, Ren L, Zhao J, Hu Y, Zhang L, Fan G, Xu J, Gu X, Cheng Z, Yu T, Xia J, Wei Y, Wu W, Xie X, Yin W, Li H, Liu M, Xiao Y, Gao H, Guo L, Xie J, Wang G, Jiang R, Gao Z, Jin Q, Wang J, Cao B. Clinical features of patients infected with 2019 novel coronavirus in Wuhan. China Lancet. 2020;395(10223):497-506.

7. Coimbra R, Edwards S, Kurihara H, Bass GA, Balogh ZJ, Tilsed J, Faccincani R, Carlucci M, Martínez Casas I, Gaarder C, Tabuenca A, Coimbra BC, Marzi I. European Society of Trauma and Emergency Surgery (ESTES) recommendations for trauma and emergency surgery preparation during times of COVID-19 infection. Eur J Trauma EmergSurg. 2020;46(3):505-10.

8. Akdur A, Karakaya E, Ayvazoglu Soy EH, Alshalabi O, Kirnap M, Arslan H, Ulubay G, Hekimoglu K, Moray G, Haberal M. Coronavirus disease (COVID-19) in kidney and liver transplant patients: a singlecenter experience. Exp Clin Transplant. 2020;18(3):270-4.

9. Boettler T, Newsome PN, Mondelli MU, Maticic M, Cordero E, Cornberg M, Berg T. Care of patients with liver disease during the COVID-19 pandemic: EASL-ESCMID position paper. JHEP Rep. 2020;2(3):100113.

10. Huang J, Lin H, Wu Y, Fang Y, Kumar R, Chen G, Lin S. COVID19 in posttransplant patients-report of 2 cases. Am J Transplant. 2020;20(7):1879-81.

11. Arslan H, Musabak U, Ayvazoglu Soy EH, Kurt Azap O, Sayin B, Akcay S, Haberal KM, Akdur A, Yildirim S, Haberal M. Incidence and immunologic analysis of coronavirus disease (COVID-19) in hemodialysis patients: a single-center experience. Exp Clin Transplant. 2020;18(3):275-283.

12. Seminari E, Colaneri M, Sambo M, Gallazzi I, Di Matteo A, Roda S, Bruno R. COVID19 IRCCS San Matteo Pavia Task Force. SARS Cov-2 infection in a renal-transplanted patient: a case report. Am J Transplant. 2020;20(7):1882-1884

13. Sahin TT, Akbulut S, Yilmaz S. COVID-19 pandemic: its impact on liver disease and liver transplantation. World J Gastroenterol. 2020;26(22):2987-99.

Publisher's Note Springer Nature remains neutral with regard to jurisdictional claims in published maps and institutional affiliations. 